\title{
A New Solution for D2D Assisted 5G AR Communications
}

Ugljesa Urosevic ( $\nabla$ ugljesa.urosevic@ucg.ac.me)

University of Montenegro https://orcid.org/0000-0002-0392-4255

\section{Zoran Veljovic}

University of Montenegro

\section{Research Article}

Keywords: BER, D2D communications, relaying, spectral efficiency

Posted Date: June 17th, 2021

DOI: https://doi.org/10.21203/rs.3.rs-507113/v1

License: (c) (1) This work is licensed under a Creative Commons Attribution 4.0 International License. Read Full License 


\section{A new solution for D2D assisted 5G AR communications}

Ugljesa Urosevic ${ }^{*}$, University of Montenegro, Faculty of Science and Mathematics,

email: ugljesa.urosevic@ucg.ac.me

Phone: +38220245839

Fax : +38220245873

Zoran Veljovic, University of Montenegro, Faculty of Electrical Engineering,

${ }^{*}$ Corresponding author 


\begin{abstract}
Device-to-Device (D2D) communications are one of the main drivers of new wireless standards. D2D improves resource utilization, spectral efficiency, energy efficiency and cellular coverage of wireless networks. In some applications like online gaming, video streaming and multimedia downloading, performances can be deteriorated for users at cell edges. This is particulary emphasises in the case of augmented reality (AR) and virtual reality (VR) technologies. AR is a highly visual, interactive method of presenting relevant digital information in the context of the physical environment, e.g. connecting employees and improving business outcomes. In this paper, a new communication scheme with D2D assistance is proposed, which can significantly increase the spectral/energy efficiency in 5G AR use cases. The proposed scheme combines multiple input multiple output (MIMO) techniques, relaying and spatial modulation (SM). It allows the formation of virtual MIMO unicast, SM multicast, and SM unicast channels between AR devices. The simulation results show that spectral/energy efficiency can be significantly increased without significantly impairing bit error rate (BER) performance.
\end{abstract}

\title{
Keywords
}

BER, D2D communications, relaying, spectral efficiency

\section{Introduction}

It is expected that over 6 billion people and over 50 billion devices will be connected in the near future. That's essentially everyone and everything connected, across world, supporting every application from consumer ultrabroadband, mobile gaming, augmented reality (AR), virtual reality (VR), and autonomous connected vehicles to global business networks, ships, planes, and agriculture connected farms. Supporting such connectivity is very challenging for many current communications systems. This fact is driving the development of the new fifth generation $(5 \mathrm{G})$ networking architecture. The $5 \mathrm{G}$ networks bring ultra-broadband speeds, exponentially higher spectral and energy efficiencies, massive scalability, significantly lower costs for mobile and fixed networks, and ultra-low latency applications such as connected vehicle, device-to-device (D2D), massive machine-to-machine (M2M) and internet of things (IoT) applications. With the development of LTE-pro and 5G standards, D2D as subsystems are being developed in parallel. In a D2D wireless communication, two nearby users can communicate 
directly with limited or no participation of the base station. D2D has the potential to support high-data rate and low latency applications effectively. D2D improves resource utilization, spectral efficiency, energy efficiency and cellular coverage of wireless networks. Due to these features, it has been envisioned as one of key technology for the 5G communication systems. AR technology can be used to visualize data from hundreds of sensors simultaneously, overlaying relevant and actionable information over environment through a headset, and D2D can make AR technology much faster, with much more data flow resulting with easier and more accessible use, for a variety of different applications, [1]-[7].

In some AR applications that include video gaming, video streaming and multimedia downloading, performances can be deteriorated as a result of insufficient spectral efficiency and not enough decreased latency.

In this paper, a new transmission scheme with D2D assistance is proposed. The assumption is that there are use cases that involve a cluster of AR users, where source node $(\mathrm{SN})$ has multicast streaming for surrounding near nodes $(\mathrm{NN})$, and unicast stream for one far node (FN), for which the direct link is negligible. In this scenario one surrounding user behaves as relay node $(\mathrm{RN})$ between $\mathrm{SN}$ and FN, i.e. it is used for improving the transmission capacity and coverage. Since the AR applications require high transmission speeds and as little delay as possible, in the proposed transmission scheme spatial modulation (SM) is implemented at both hops. SM can increase spectral/energy efficiency, because part of the data can be indirectly transmitted by detecting active antennas, [8][9]. In the proposed scheme, the number of antennas at SN and RN can be arbitrary, but only two antennas are active at each slot, which means that only two radio frequency (RF) chains are needed, i.e. lower costs can be achieved. Advanced communication paradigm including relaying and multiple-input and multiple-output (MIMO) can be used to enhance the capacity and reliability performance of the system or network with reduced complexity [10]-[12]. In MIMO, multiple antennas transmit and receive at the same frequency/time which results in a capacity gain. However, the performance of MIMO systems depend on antenna spacing, synchronization among the transmit antennas and the interference cancellation techniques used to reduce the inter-channel interference (ICI) at the receiver. Unlike MIMO, SM can allow only one antenna to transmit at a particular time instant while others antennas remain silent. This in turn reduces ICI and interference. SM-MIMO offers reduced receiver complexity. Further, relay techniques can be used in combination with MIMO to create distributed MIMO channels, [13]-[17]. In this way, it is possible to form distributed MIMO systems that have more virtual transmitting and receiving 
antennas than any of the nodes in the distributed scheme itself. Virtual antenna technology can nicely deal with the problem of lacking multiple antennas of terminals.

In this paper, we considered scenarios where there is at least one AR NN user with direct link to FN. A new signal processing at a $\mathrm{RN}$ is proposed, which is amplified and forward (AF) in a manner, to avoid decoding. This is especially important in terms of energy savings at RN, because decode and forward (DF) process can significantly complicate processing, especially for higher order modulations. Limited battery capacity of mobile devices is a barrier to fully exploiting the benefits of D2D communication. Meanwhile, high data rate D2D communication is required to support the increasing traffic demand of emerging applications.

The proposed scheme creates $4 \times X$ virtual MIMO/quasi orthogonal space time block code (QOSTBC) unicast channel between SN and FN. Also, new proposed processing aims to emulate distributed full orthogonal STBC (OSTBC), but the code rate remains the same as for the classical QOSTBC. SM multicast channels are formed between SN and NNs, while SM unicast channel between RN and FN is also formed.

Here, a detailed description of the distributed coding procedure for the proposed scheme is presented. The simulation results show that the proposed scheme provides a possible trade-off between bit error rate (BER) and spectral/energy efficiency.

This paper is composed in the following order. In Sect. 2 the proposed scheme is described, while the simulation results are presented in Sect. 3. Conclusions are drawn in Sect. 4.

\section{The proposed AR transmission scheme with D2D assistance}

The AR D2D assisted transmission scenario is presented in Figure 1. It is assumed that AR SN has unicast stream for one AR FN. Furthermore, there exists one AR RN between SN and FN. SN is equipped with $T$ transmitting antennas, RN with $N$ antennas, while NNs and FN are equipped with $R$ receiving antennas. Spatial modulation is implemented at SN, as well at RN. At source node SM is used for multicast streaming towards surrounding NNs and RN. Also, new distributed MIMO/STBC scheme between SN and FN is proposed in this paper, so SN transmits unicast stream towards FN via virtual MIMO channel. The proposed processing aims to emulate full orthogonal STBC regarding BER, and QOSTBC in terms of code rate. In parallel with that, RN can indirectly convey another unicast stream towards FN. Simply put, SN has unicast data for FN, and multicast data for surrounding NNs, while 
RN enables virtual MIMO channel between SN and FN. Also RN has its own unicast data for the same FN. It is assumed that link between SN and FN is completely degraded.

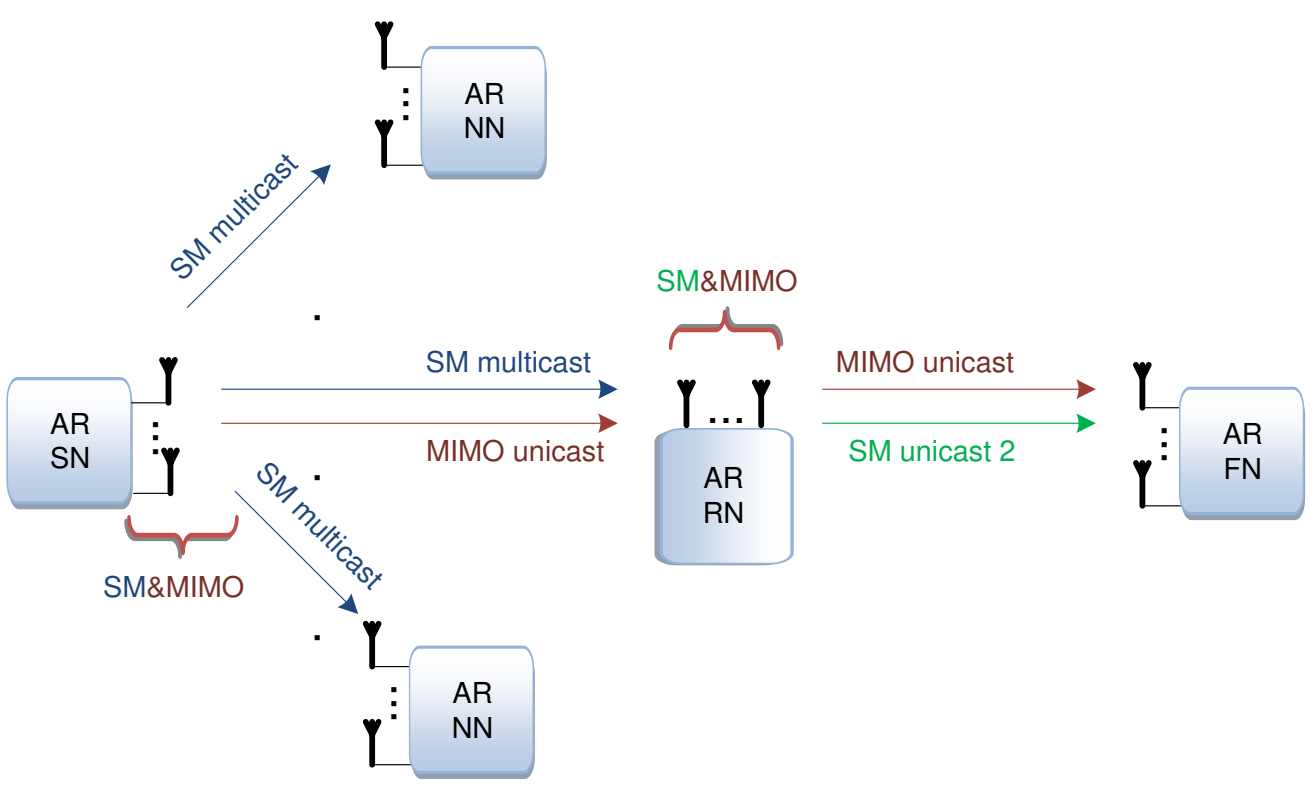

Fig. 1. AR D2D assisted transmission scenario.

In the first hop, multicast streams are indirectly transmitted i.e. additional spectral/time/energy resources are not occupied, while in the second hop unicast stream originated from RN is also transmitted with "zero cost" in terms of available spectral/time/energy resources.

There is a cluster of nodes, e.g. cluster that acts in the form of an AR experience, which in itself entails the need for higher data rates and lower delays. Also an important parameter is the complexity of data processing on the devices themselves as well as energy efficiency. It is always desirable to have a longer battery life.

SN uses SM technique, and there are $T$ available transmitting antennas. Bits from multicast stream are used for determining which antennas will be active at the SN. It is assumed that two antennas are active at each time slot. Those bits that determine the active antennas are not transmitted directly, but are decoded on the destination based on detecting which antennas are, from the possible set of antennas, used during transmission.

Unicast bits are modulated according to a certain modulation scheme and then based on the new proposed schemes are transmitted from the selected SN antennas, in order to create virtual MIMO channels between SN and FN. 
In the proposed scheme, two SN antennas are active at each time slot, which means that number of possible combinations depends on the total number of antennas. If $\mathrm{SN}$ is equipped with only two antennas, it means that in addition to virtual MIMO, SM isn’t implemented, i.e. only unicast bits are transmitted. If the number of antennas is 3 , it means that three pairs of two active antennas are possible, i.e. $T_{1}-T_{2}, T_{1}-T_{3}$ and $T_{2}-T_{3}$. Three antenna combinations require at least two bits to select active antenna pairs. In order to cover all bits combinations it was proposed that one pair $\left(T_{1}-T_{2}\right)$ antennas has two possible transmit power states $P_{1}$ and $P_{2}$. Table 1 shows the mapping of multicast bits for antenna selection, and combinations of active antennas and transmit powers.

Table 1. One possible mapping of selecting (multicast) bits and combinations of active antennas and transmit powers,

$$
T=3
$$

\begin{tabular}{ccc}
\hline Bits & Active antenna pairs & Transmit power \\
\hline 00 & $T_{1}-T_{2}$ & $P_{1}$ \\
01 & $T_{1}-T_{2}$ & $P_{2}$ \\
10 & $T_{1}-T_{3}$ & $P_{1}$ \\
11 & $T_{2}-T_{3}$ & $P_{1}$ \\
\hline
\end{tabular}

If the number of antennas is 4 , it means that six different pairs of two active antennas are possible. Three antenna combinations require at least three bits to select antenna pairs. In order to cover all combinations with three bits, it was proposed that two antenna pairs have two possible emission states. Table 2 shows the mapping of selecting bits and combinations of active antennas and emission powers for $T=4$, while table 3 shows the mapping of selecting bits and combinations of active antennas and emission powers for $T=5$.

Table 2. One possible combinations of selecting bits and combinations of active antennas and transmit powers, $T=4$

\begin{tabular}{ccc}
\hline Bits & Active antenna pairs & Transmit power \\
\hline 000 & $T_{1}-T_{2}$ & $P_{1}$ \\
001 & $T_{1}-T_{2}$ & $P_{2}$ \\
010 & $T_{1}-T_{3}$ & $P_{1}$ \\
011 & $T_{1}-T_{3}$ & $P_{2}$ \\
\hline
\end{tabular}




\begin{tabular}{ccc}
\hline $\mathbf{1 0 0}$ & $T_{1}-T_{4}$ & $P_{1}$ \\
\hline $\mathbf{1 0 1}$ & $T_{2}-T_{3}$ & $P_{1}$ \\
110 & $T_{2}-T_{4}$ & $P_{1}$ \\
11 & $T_{3}-T_{4}$ & $P_{1}$ \\
\hline
\end{tabular}

Table 3. One possible combinations of selecting bits and combinations of active antennas and transmit powers, $T=5$

\begin{tabular}{ccc}
\hline Bits & Active antenna pairs & Transmit power \\
\hline $\mathbf{0 0 0 0}$ & $T_{1}-T_{2}$ & $P_{1}$ \\
\hline $\mathbf{0 0 0 1}$ & $T_{1}-T_{2}$ & $P_{2}$ \\
$\mathbf{0 0 1 0}$ & $T_{1}-T_{3}$ & $P_{1}$ \\
$\mathbf{0 0 1 1}$ & $T_{1}-T_{3}$ & $P_{2}$ \\
$\mathbf{0 1 0 0}$ & $T_{1}-T_{4}$ & $P_{1}$ \\
$\mathbf{0 1 0 1}$ & $T_{1}-T_{4}$ & $P_{2}$ \\
$\mathbf{0 1 1 0}$ & $T_{1}-T_{5}$ & $P_{1}$ \\
$\mathbf{0 1 1 1}$ & $T_{1}-T_{5}$ & $P_{2}$ \\
$\mathbf{1 0 0 0}$ & $T_{2}-T_{3}$ & $P_{1}$ \\
\hline $\mathbf{1 0 0 1}$ & $T_{2}-T_{3}$ & $P_{2}$ \\
\hline $\mathbf{1 0 1 0}$ & $T_{2}-T_{4}$ & $P_{1}$ \\
\hline $\mathbf{1 0 1 1}$ & $T_{2}-T_{4}$ & $P_{1}$ \\
\hline $\mathbf{1 1 0 0}$ & $T_{2}-T_{5}$ & $P_{2}$ \\
\hline $\mathbf{1 1 0 1}$ & $T_{3}-T_{4}$ & $P_{1}$ \\
\hline & $T_{3}-T_{5}$ & $T_{4}-T_{5}$ \\
\hline
\end{tabular}

Here we proposed the new distributed space time block code (STBC) scheme that is originally dedicated for classical MIMO systems with four transmit antennas. The goal is to keep code rate of QOSTBC and BER performance of OSTBC. The new scheme enables virtual $4 \times X$ MIMO channel. 
The assumption is that channel remains the same during the four time slots. Therefore, for every four unicast STBC symbols, i.e. four time slots, depending on the number of antennas at the $\mathrm{SN}$, a part of multicast bits is used for selecting active antennas.

Transmitted symbol sequences from two, $i$-th and $j$-th, selected SN antennas are:

$\mathbf{S N}_{i}=\frac{P_{s e l}}{\sqrt{2}}\left[\begin{array}{llll}a_{1} & -a_{2}^{*} & -a_{3}^{*} & a_{4}\end{array}\right]$

$\mathbf{S N}_{j}=\frac{P_{s e l}}{\sqrt{2}}\left[\begin{array}{llll}a_{2} & a_{1}^{*} & -a_{4}^{*} & -a_{3}\end{array}\right]$

where $P_{\text {sel }}=P_{1}$ or $P_{\text {sel }}=P_{2}$ according to mapping of selecting bits and power states.

For every four time slots, a part of RN unicast bits (unicast stream 2) is used for selecting active antennas. That procedure is analog to mapping at $\mathrm{SN}$.

The received signal matrices at $k$-th and $l$-th $\mathrm{RN}$ active antenna at four time slots are given with:

$$
\begin{aligned}
& \mathbf{R N}_{k_{-} \text {rec }}=\mathbf{S N}_{i} \cdot h_{R-S_{-} k, i}+\mathbf{S N}_{j} \cdot h_{R-S_{-} k, j}+\mathbf{n}_{A W G N_{-} R N_{-} k} \\
& \mathbf{R N}_{l_{-} \text {rec }}=\mathbf{S N}_{i} \cdot h_{R_{-} S_{-} l, i}+\mathbf{S} \mathbf{N}_{j} \cdot h_{R-S_{-} l, j}+\mathbf{n}_{A W G N_{-} R N_{-} l}
\end{aligned}
$$

where $h_{R-S_{-} k, i}, h_{R-S_{-} k, j}, h_{R-S_{-} l, i}$ and $h_{R-S_{-} l, j}$ denote the radio channel coefficients between the active $k$-th and $l$ th $\mathrm{RN}$ receiving antennas and the active $i$-th and $j$-th, $\mathrm{SN}$ transmitting antennas, while $\mathbf{n}_{A W G N_{-} R N_{-} k}$ and $\mathbf{n}_{A W G N_{-} R N_{-} l}$ are additive white Gaussian noise (AWGN) matrixes at $k$-th and $l$-th RN antenna.

In order to reduce the processing complexity at $\mathrm{RN}$ as well as the energy consumption new processing is performed in AF manner. There is no STBC decoding at RN. For higher order modulations this can be very time consuming. The proposed signal processing at the RN allows distributed STBC with low complexity, so that the RN is essentially transparent for FN in terms of unicast stream from SN. 
RN performs the proposed process of determining from which SN antennas the symbols are sent. Let $\left|\mathbf{M}_{s}\right|$ denote the set of different modules of transmitted constellation points, i.e. $s=1+1$ for 4QAM (consequence of two possible transmitting power states), $s=2+1$ for 8QAM, etc.

Deciding comes down to looking for the minimum of the next set:

$\min _{\substack{\forall s \\ \forall i \\ \forall j}}\left\{\left|\mathbf{M}_{s}\right|-\left|\frac{\left.\mid \begin{array}{ll}\tilde{h}_{R-S_{-} k, i}^{*} & \tilde{h}_{R-S_{-} k, j}\end{array}\right] \cdot\left[\begin{array}{ll}\mathbf{R N}_{k-r e c}(1) & \mathbf{R N}_{k_{-} r e c}^{*}(1)\end{array}\right]^{\mathrm{T}}}{\left|\tilde{h}_{R-S_{-} k, i}\right|^{2}+\left|\tilde{h}_{R-S_{-} k, j}\right|^{2}}\right|+\right.$

$\left|\mathbf{M}_{s}\right|-\left|\frac{\left.\mid \begin{array}{ll}\tilde{h}_{R-S_{-} k, j}^{*} & -\tilde{h}_{R-S_{-} k, i}\end{array}\right] \cdot\left[\begin{array}{ll}\mathbf{R N}_{k-r e c}(1) & \mathbf{R N}_{k_{-} r e c}^{*}(1)\end{array}\right]^{\mathrm{T}}}{\left|\tilde{h}_{R-S_{-} k, i}\right|^{2}+\left|\tilde{h}_{R-S_{-} k, j}\right|^{2}}\right|+$

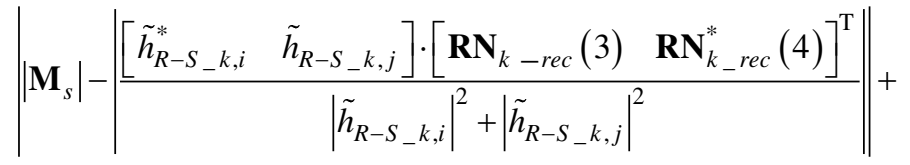

|| $\mathbf{M}_{s}|-| \frac{\left[\begin{array}{ll}\tilde{h}_{R-S_{-} k, j}^{*} & -\tilde{h}_{R-S_{-} k, i}\end{array}\right] \cdot\left[\begin{array}{ll}\mathbf{R N}_{k-r e c}(3) & \mathbf{R N}_{k_{-} r e c}^{*}(4)\end{array}\right]^{\mathrm{T}}}{\left|\tilde{h}_{R-S_{-} k, i}\right|^{2}+\left|\tilde{h}_{R-S_{-} k, j}\right|^{2}} \mid+$

|| $\mathbf{M}_{s}|-| \frac{\left[\begin{array}{ll}\tilde{h}_{R-S_{-} l, i}^{*} & \tilde{h}_{R-S_{-} l, j}\end{array}\right] \cdot\left[\begin{array}{ll}\mathbf{R} \mathbf{N}_{l-r e c}(1) & \mathbf{R N}_{l_{-} \text {rec }}^{*}(1)\end{array}\right]^{\mathrm{T}}}{\left|\tilde{h}_{R-S_{-} l, i}\right|^{2}+\left|\tilde{h}_{R-S_{-} l, j}\right|^{2}} \mid+$

$\left|\mathbf{M}_{s}\right|-\left|\frac{\left[\begin{array}{ll}\tilde{h}_{R-S_{-} l, j}^{*} & -\tilde{h}_{R-S_{-} l, i}\end{array}\right] \cdot\left[\begin{array}{ll}\mathbf{R N}_{l-r e c}(1) & \mathbf{R N}_{l_{-} r e c}^{*}(1)\end{array}\right]^{\mathrm{T}}}{\left|\tilde{h}_{R-S_{-} l, i}\right|^{2}+\left|\tilde{h}_{R-S_{-} l, j}\right|^{2}}\right|+$

$\left|\mathbf{M}_{s}\right|-\left|\frac{\left[\begin{array}{ll}\tilde{h}_{R-S_{-} l, i}^{*} & \tilde{h}_{R-S_{-} l, j}\end{array}\right] \cdot\left[\begin{array}{ll}\mathbf{R N}_{l-r e c}(3) & \mathbf{R N}_{l_{-} r e c}^{*}(4)\end{array}\right]^{\mathrm{T}}}{\left|\tilde{h}_{R-S_{-} l, i}\right|^{\mathrm{T}}+\left|\tilde{h}_{R-S_{-} l, j}\right|^{2}}\right|+$

$$
\left.|| \mathbf{M}_{s}|-| \frac{\left[\begin{array}{ll}
\tilde{h}_{R-S_{-} l, j}^{*} & -\tilde{h}_{R-S_{-} l, i}
\end{array}\right] \cdot\left[\begin{array}{ll}
\mathbf{R N}_{l-\text { rec }}(3) & \mathbf{R N}_{l_{-} \text {rec }}^{*}(4)
\end{array}\right]^{\mathrm{T}}}{\left|\tilde{h}_{R-S_{-} l, i}\right|^{2}+\left|\tilde{h}_{R-S_{-}, j}\right|^{2}}||\right\}
$$


where $\tilde{h}_{R-S_{-} k, i}, \tilde{h}_{R-S_{-} k, j}, \tilde{h}_{R-S_{-} l, i}$, and $\tilde{h}_{R-S_{-} l, j}$ are estimated channel gains between $k$-th and $l$-th RN antenna and $i$-th and $j$-th SN antennas. Based on detected active antennas at SN i.e. $\tilde{i}$ and $\tilde{j}, \mathrm{RN}$ forwards estimated channels coefficients $\tilde{h}_{R-S_{-} k, \tilde{i}}, \tilde{h}_{R-S_{-} k, \tilde{j}}, \tilde{h}_{R-S_{-} l, \tilde{i}}$, and $\tilde{h}_{R-S_{-} l, \tilde{j}}$ to the FN.

RN performs new proposed processing in order to create virtual MIMO/STBC channel, and simultaneously to decrease inter-symbol interference caused by lack of full orthogonality between QOSTBC symbols. It transmits the next symbols at four time slots:

$$
\begin{aligned}
& \mathbf{R}_{k_{-} \text {trans }}(1)=\frac{1}{\sqrt{2}} \mathbf{R}_{k_{-} r e c}(1)+\frac{I_{1}}{2 \tilde{g}_{F-R_{-} r, k}} \\
& \mathbf{R}_{k_{-} \text {trans }}(2)=\frac{1}{\sqrt{2}} \mathbf{R}_{k_{-} r e c}(2)+\frac{I_{2}}{2 \tilde{g}_{F-R_{-} r, k}} \\
& \mathbf{R}_{k_{-} \text {trans }}(3)=\frac{1}{\sqrt{2}} \mathbf{R}_{k_{-} r e c}(3)+\frac{I_{3}}{2 \tilde{g}_{F-R_{-} r, k}} \\
& \mathbf{R}_{k_{-} \text {trans }}(4)=\frac{1}{\sqrt{2}} \mathbf{R}_{k_{-} r e c}(4)+\frac{I_{4}}{2 \tilde{g}_{F-R_{-} r, k}} \\
& \mathbf{R}_{l_{-} \text {trans }}(1)=-\frac{1}{\sqrt{2}} \mathbf{R N}_{l_{-} r e c}^{*}(3)+\frac{I_{1}}{2 \tilde{g}_{F-R_{-} r, l}} \\
& \mathbf{R}_{l_{-} \text {trans }}(2)=-\frac{1}{\sqrt{2}} \mathbf{R N}_{l_{-} r e c}^{*}(4)+\frac{I_{2}}{2 \tilde{g}_{F-R_{-} r, l}} \\
& \mathbf{R N}_{l_{-} \text {trans }}(3)=\frac{1}{\sqrt{2}} \mathbf{R}_{l_{-} r e c}^{*}(1)+\frac{I_{3}}{2 \tilde{g}_{F-R_{-} r, l}} \\
& \mathbf{R}_{l_{-} \text {trans }}(4)=\frac{1}{\sqrt{2}} \mathbf{R N}_{l_{-} r e c}^{*}(2)+\frac{I_{4}}{2 \tilde{g}_{F-R_{-} r, l}}
\end{aligned}
$$

where $\tilde{g}_{F-R_{-} r, k}$ and $\tilde{g}_{F-R_{-}, r, l}$ denote estimated channel gains between $r$-th, $r=1, \ldots, R$, FN receiving antenna and RN active antennas, while: 
$\left[\begin{array}{l}I_{1} \\ I_{2} \\ I_{3} \\ I_{4}\end{array}\right]=\left[\begin{array}{llll}\tilde{h}_{R-S_{-} k, \tilde{i}} \cdot \tilde{g}_{F-R_{-} r, k} & \tilde{h}_{R-S_{-} k, \tilde{j}} \cdot \tilde{g}_{F-R_{-} r, k} & \tilde{h}_{R-S_{-} l, \tilde{i}}^{*} \cdot \tilde{g}_{F-R_{-} r, l} & \tilde{h}_{R-S_{-} l, \tilde{j}}^{*} \cdot \tilde{g}_{F-R_{-} r, l} \\ \tilde{h}_{R-S_{-} k, \tilde{j}}^{*} \cdot \tilde{g}_{F-R_{-} r, k}^{*} & -\tilde{h}_{R-S_{-} k, \tilde{i}}^{*} \cdot \tilde{g}_{F-R_{-} r, k}^{*} & \tilde{h}_{R-S_{-} l, \tilde{j}} \cdot \tilde{g}_{F-R_{-} r, l}^{*} & -\tilde{h}_{R-S_{-} l, \tilde{i}} \cdot \tilde{g}_{F-R_{-} r, l}^{*} \\ \tilde{h}_{R-S_{-} l, \tilde{i}} \cdot \tilde{g}_{F-R_{-} r, l}^{*} & \tilde{h}_{R-S_{-} l, \tilde{j}} \cdot \tilde{g}_{F-R_{-} r, l}^{*} & -\tilde{h}_{R-S_{-} k, \tilde{i}}^{*} \cdot \tilde{g}_{F-R_{-} r, k}^{*} & -\tilde{h}_{R-S_{-} k, \tilde{j}}^{*} \cdot \tilde{g}_{F-R_{-} r, k}^{*} \\ \tilde{h}_{R-S_{-} l, \tilde{j}}^{*} \cdot \tilde{g}_{F-R_{-} r, l} & -\tilde{h}_{R-S_{-} l, \tilde{i}}^{*} \cdot \tilde{g}_{F-R_{-} r, l} & -\tilde{h}_{R-S_{-} k, \tilde{j}} \cdot \tilde{g}_{F-R_{-} r, k} & \tilde{h}_{R-S_{-} k, \tilde{i}} \cdot \tilde{g}_{F-R_{-} r, k}\end{array}\right]^{-1} \cdot\left[\begin{array}{c}-K \cdot S_{4} \\ K \cdot S_{3} \\ K \cdot S_{2} \\ -K \cdot S_{1}\end{array}\right]$

Here,

$S_{1}=\frac{1}{2}\left(\frac{\mathbf{R N}_{k_{-} r e c}(1) \cdot h_{R_{-} S_{-} k, \tilde{i}}^{*}+\mathbf{R N}_{k_{-} r e c}^{*}(2) \cdot h_{R_{-} S_{-} k, \tilde{j}}}{\left|h_{R-S_{-} k, \tilde{i}}\right|^{2}+\left|h_{R_{-} S_{-} k, \tilde{j}}\right|^{2}}+\frac{\mathbf{R N}_{l_{-} r e c}(1) \cdot h_{R-S_{-} l, \tilde{i}}^{*}+\mathbf{R N}_{l_{-} r e c}^{*}(2) \cdot h_{R-S_{-} l, \tilde{j}}}{\left|h_{R-S_{-} l, \tilde{i}}\right|^{2}+\left|h_{R_{-} S_{-} l, \tilde{j}}\right|^{2}}\right)$

$S_{2}=\frac{1}{2}\left(\frac{\mathbf{R N}_{k_{-} r e c}(1) \cdot h_{R-S_{-} k, \tilde{j}}^{*}+\mathbf{R N}_{k_{-} r e c}^{*}(2) \cdot h_{R-S_{-} k, \tilde{i}}}{\left|h_{R-S_{-} k, \tilde{i}}\right|^{2}+\left|h_{R_{-} S_{-} k, \tilde{j}}\right|^{2}}+\frac{\mathbf{R N}_{l_{-} r e c}(1) \cdot h_{R-S_{-} l, \tilde{j}}^{*}+\mathbf{R N}_{l_{-} r e c}^{*}(2) \cdot h_{R-S_{-} l, \tilde{i}}}{\left|h_{R-S_{-} l, \tilde{i}}\right|^{2}+\left|h_{R_{-} S_{-} l, \tilde{j}}\right|^{2}}\right)$

$S_{3}=\frac{1}{2}\left(\frac{\mathbf{R N}_{k_{-} r e c}^{*}(3) \cdot h_{R_{-} S_{-} k, \tilde{i}}+\mathbf{R N}_{k_{-} r e c}(4) \cdot h_{R-S_{-} k, \tilde{j}}^{*}}{\left|h_{R-S_{-} k, \tilde{i}}\right|^{2}+\left|h_{R_{-} S_{-} k, \tilde{j}}\right|^{2}}+\frac{\mathbf{R N}_{l_{-} r e c}^{*}(3) \cdot h_{R-S_{-} l, \tilde{i}}+\mathbf{R N}_{l_{-} r e c}(4) \cdot h_{R-S_{-} l, \tilde{j}}^{*}}{\left|h_{R-S_{-} l, \tilde{i}}\right|^{2}+\left|h_{R_{-} S_{-} l, \tilde{j}}\right|^{2}}\right)$

$S_{4}=\frac{1}{2}\left(\frac{\mathbf{R N}_{k_{-} r e c}^{*}(1) \cdot h_{R-S_{-} k, \tilde{j}}+\mathbf{R N}_{k_{-} r e c}(2) \cdot h_{R-S_{-} k, \tilde{i}}^{*}}{\left|h_{R-S_{-} k, \tilde{i}}\right|^{2}+\left|h_{R-S_{-} k, \tilde{j}}\right|^{2}}+\frac{\mathbf{R N}_{l_{-} r e c}^{*}(1) \cdot h_{R-S_{-} l, \tilde{j}}+\mathbf{R N}_{l_{-} r e c}(2) \cdot h_{R-S_{-} l, \tilde{i}}^{*}}{\left|h_{R-S_{-} l, \tilde{i}}\right|^{2}+\left|h_{R_{-} S_{-} l, \tilde{j}}\right|^{2}}\right)$

$K=\frac{2 \operatorname{Re}\left\{h_{R-S_{-} k, i} \cdot g_{F-R_{-} r, k} \cdot h_{R-S_{-} l, j}^{*} \cdot g_{F-R_{-} r, l}^{*}-h_{R-S_{-} k, j} \cdot g_{F-R_{-} r, k} \cdot h_{R-S_{-} l, i}^{*} \cdot g_{F-R_{-} r, l}^{*}\right\}}{\left|h_{R_{-} S_{-} k, i} \cdot g_{F-R_{-} r, k}\right|^{2}+\left|h_{R_{-} S_{-} k, j} \cdot g_{F-R_{-} r, k}\right|^{2}+\left|h_{R-S_{-} l, i}^{*} \cdot g_{F-R_{-} r, l}\right|^{2}+\left|h_{R-S_{-} l, j}^{*} \cdot g_{F-R_{-} r, l}\right|^{2}}$

The received signal at $r$-th FN antenna is given with:

$\mathbf{F N}_{\text {rec }}=\mathbf{R N}_{k_{-} \text {trans }} \cdot g_{F-R_{-} r, k}+\mathbf{R N}_{l_{-} \text {trans }} \cdot g_{F-R_{-} r, l}+\mathbf{n}_{A W G N-F N_{-} r}$ 
where $g_{F-R_{-}, k}$ and $g_{F-R_{-} r, l}$ are channel gains between $r$-th FN antenna and RN active antennas, while $\mathbf{n}_{A W G N-F N_{-} r}$ is AWGN matrix at $r$-th FN antenna.

Based on the proposed distributed scheme a virtual $4 \times R$ MIMO channel is created between SN and FN, Figure 2 .

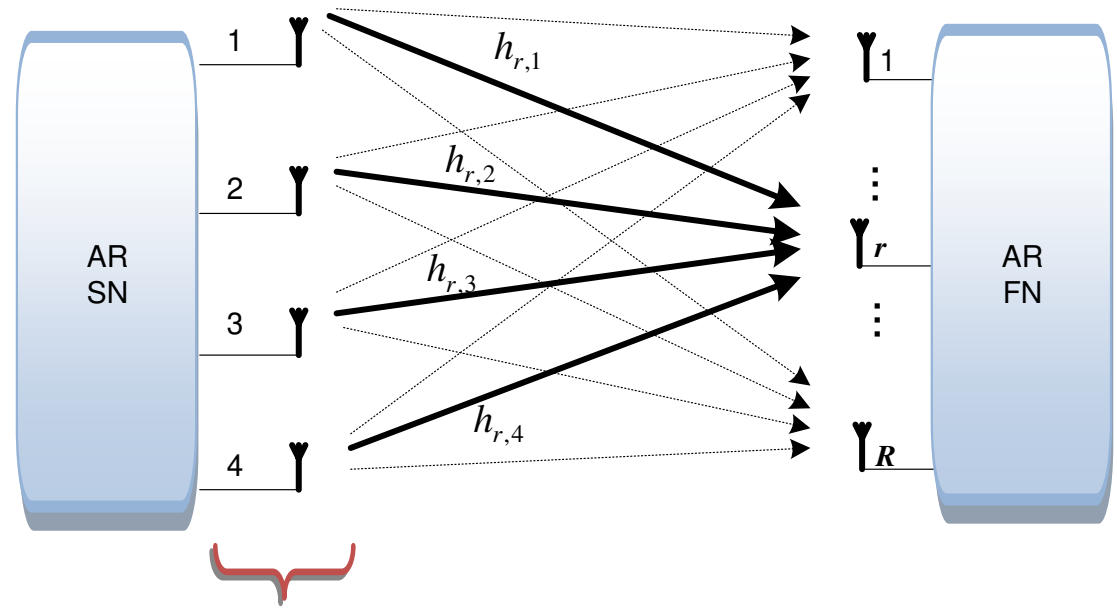

Virtual transmitting antennas

Fig. 2. Virtual $4 \times R$ MIMO channel between $\mathrm{SN}$ and FN. Bold lines represent $4 \times 1 \mathrm{MISO}$ channel between $\mathrm{SN}$ and $r$-th FN antenna

Virtual $4 \times R$ MIMO channel coefficients between $\mathrm{SN}$ and FN are given with:

$\bigcup_{r=1}^{R} h_{r, 1}=h_{R-S_{-} k, i} \cdot g_{F-R_{-} r, k}$

$\bigcup_{r=1}^{R} h_{r, 2}=h_{R-S_{-} k, j} \cdot g_{F-R_{-} r, k}$

$\bigcup_{r=1}^{R} h_{r, 3}=h_{R-S_{-} l, i}^{*} \cdot g_{F-R_{-} r, l}$

$\bigcup_{r=1}^{R} h_{r, 4}=h_{R-S_{-} l, j}^{*} \cdot g_{F-R_{-} r, l}$

Decoding of indirectly transmitted bits, i.e. unicast stream originated from RN, is performed by demapping active $\mathrm{RN}$ antennas indexes $k$ and $l$, and bits combinations. 
Looking comprehensively, virtual MIMO unicast channel is formed between SN and FN, another SM unicast channel between RN and FN, and SM channels between SN and NNs, Figure 3.

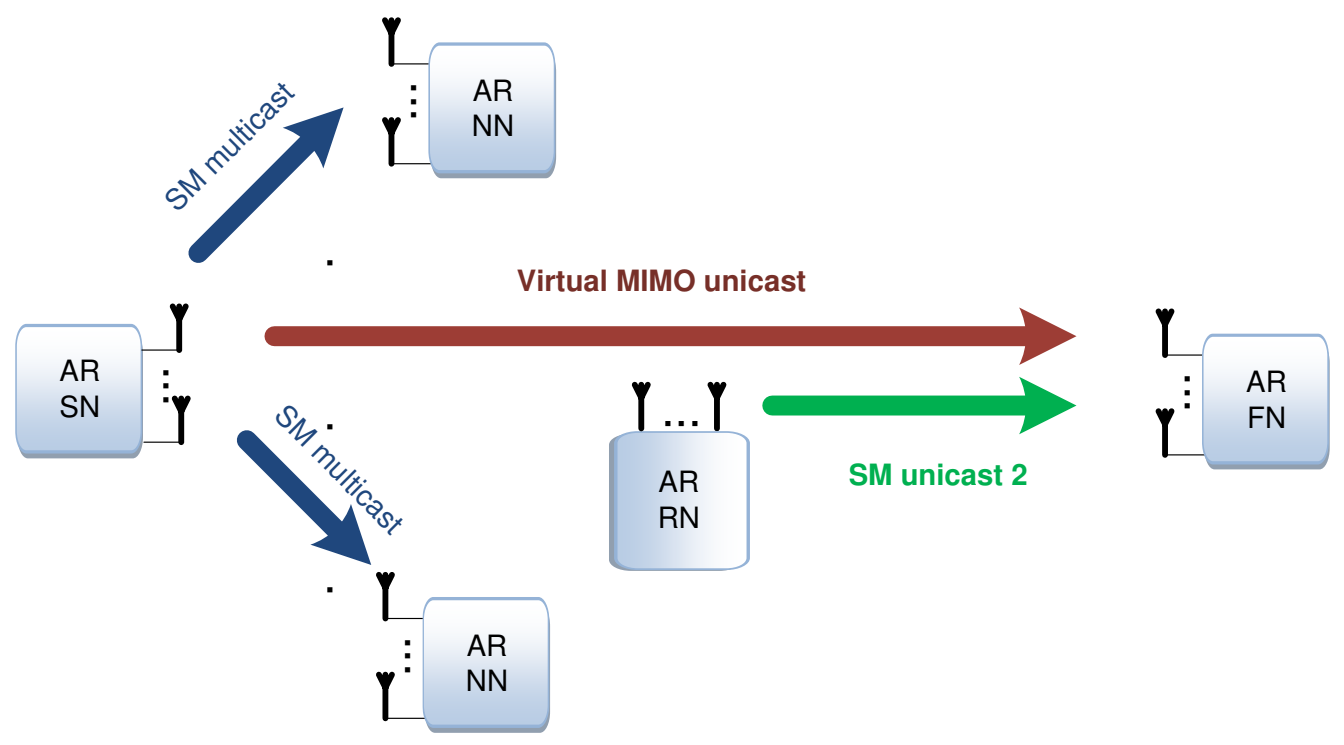

Fig. 3. Virtual MIMO unicast channel between SN and FN, another SM unicast channel between RN and FN, and SM channels between SN and NNs.

\section{Simulation results}

In this section simulation results are presented for the proposed transmission system. Multiple transmit antennas that can be selected at the SN and RN, multiple receive antennas at the FN, 4QAM modulation scheme, and Rayleigh channel conditions are considered. Monte Carlo simulations were performed for obtaining BER values. Perfect channel estimation is assumed. The graphs show the BER curves regarding signal to noise ratio (SNR) for different simulation parameters. SNR refers only to directly transmitted data, i.e. for the same energy/spectral/time unit additional bits are indirectly transmitted in the case of implemented SM. BER curves are presented in Figure 4.

Scenarios with different parameters were considered. In each of them, a virtual MIMO channel is created between SN and FN. The proposed scheme with SM-MIMO transmission was compared with the scenario without the implementation of SM. The probability of error was compared with the remark that additional SM bits are indirectly 
transmitted, i.e. the same energy/spectrum/time resources are used as in the case without the SM deployment. For the same number of time slots, more bits can be transferred for the same unit of energy/frequency bandwidth/time. In the case when the number of possible $\mathrm{SN} / \mathrm{RN}$ active antennas is 3 , that means that for every 4 time slots, i.e. for every four 4-QAM symbols, or 8 bits, two more SN multicast/RN unicast bits are conveyed in addition. When the number of SN/RN antennas is 4, an additional three SN multicast/RN unicast bits are transmitted for every eight bits, while for five antennas at the SN/RN an additional $4 \mathrm{SN}$ multicast/RN unicast bits are transmitted.

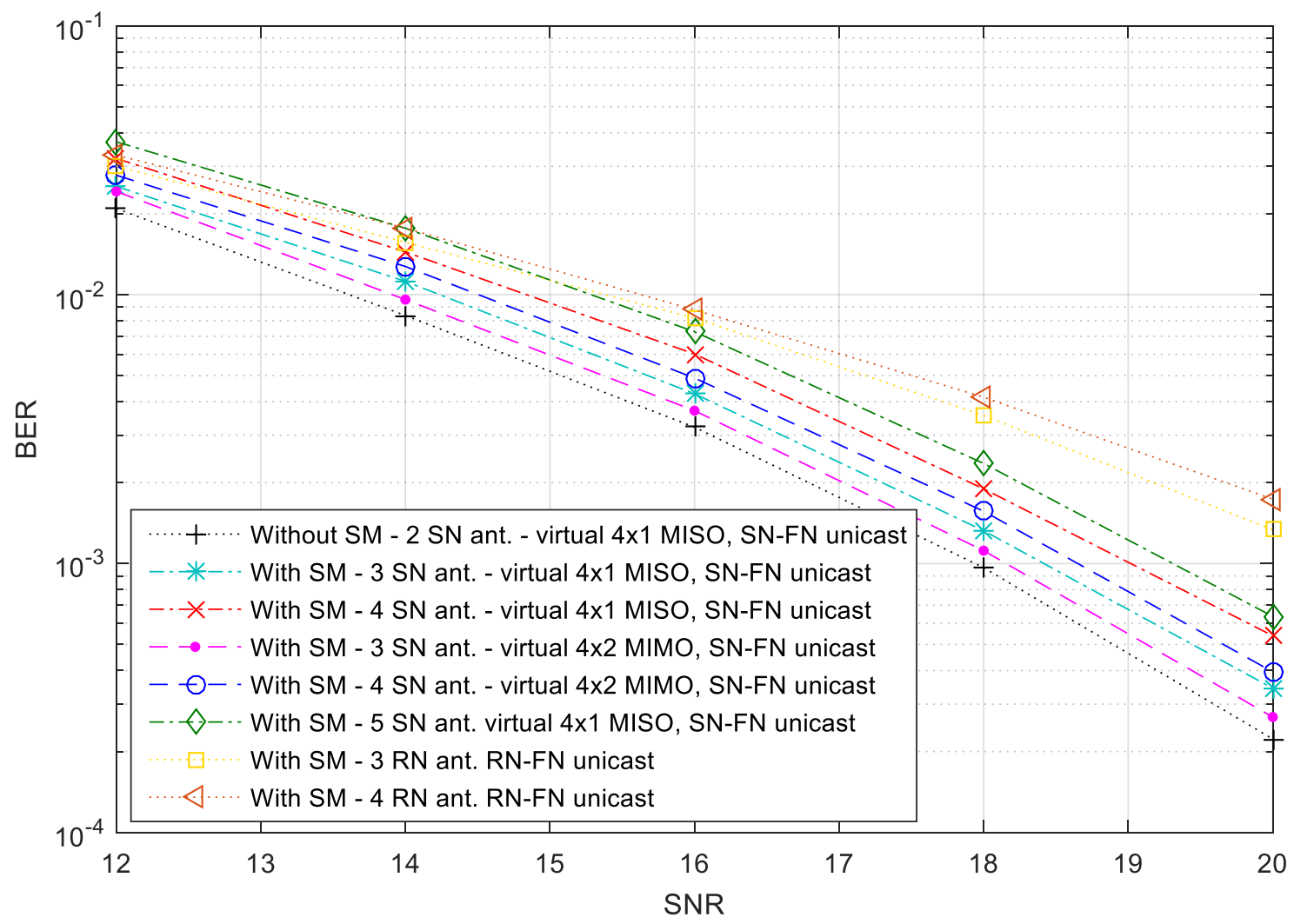

Fig. 4. BER performances of the proposed transmission scheme

In addition to the obvious energy gains, it should be noted that spectral efficiency is increased, e.g. for $25 \%, 37.5 \%$, and $50 \%$ at the each hop. In the case of the higher order modulation schemes, the increase in spectral efficiency is reduced because there are more directly transmitted bits within one time slot. In the case of 32QAM and 64QAM modulation, the corresponding increasing of spectral efficiency at each hop is $10 \%, 15 \%, 20 \%$ and $8.3 \%, 12.5 \%$, $16.6 \%$, respectively. 
Obviously, better BER values are achieved with a system without SM, however, it can be seen from the figure that for certain considered parameters in terms of the number of antennas at SN and FN degradation of BER performance is not significant. That is, for AR applications where the key factors are higher data rate, lower latency and higher spectral and energy efficiency, a trade-off can be found in relation to the error probability as a lower priority parameter. Increasing of spectral efficiency in relation to the number of SN/RN antennas and modulation orders is given in Figure 5.

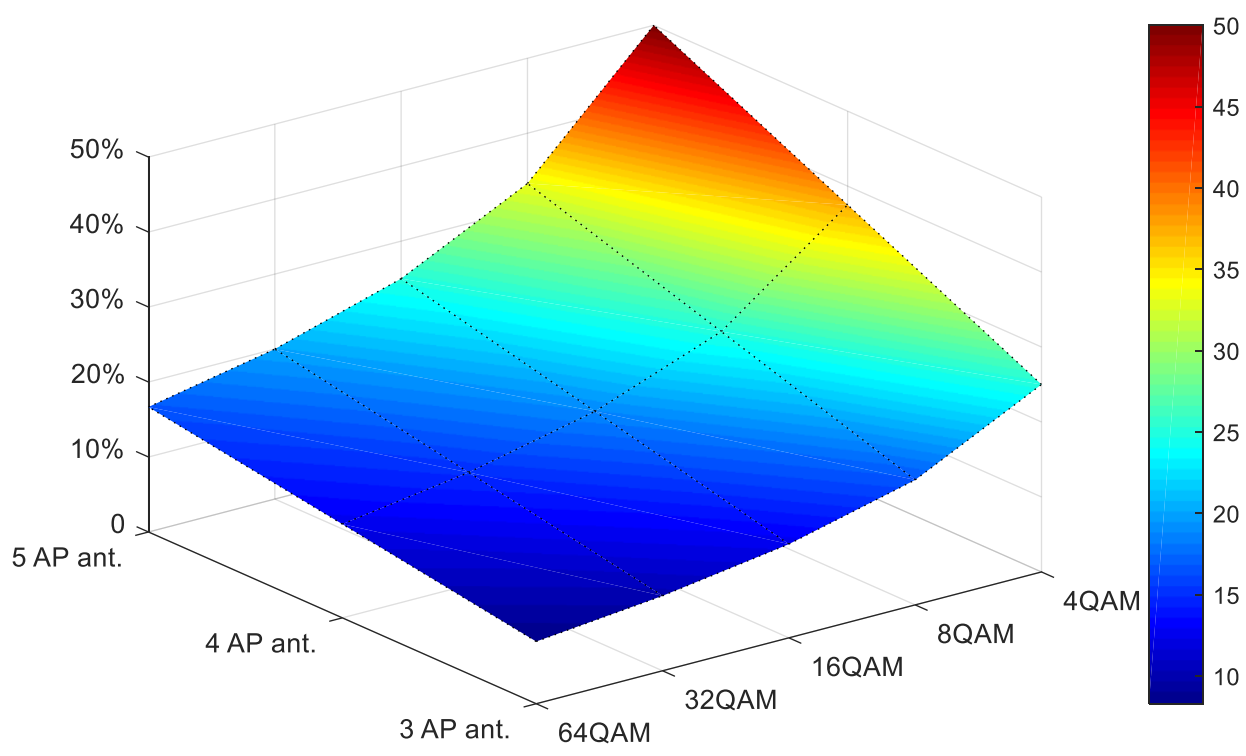

Fig. 5. Increasing of spectral efficiency in relation to the number of SN/RN antennas and modulation schemes

As it has already been said, the decoding process is not performed at $\mathrm{RN}$, but new AF-style scheme is proposed, which reduces the complexity of data processing and energy consumption, especially for higher-order modulations. Also, virtual $4 \times X$ MIMO channels are formed between $\mathrm{SN}$ and $\mathrm{FN}$, while a maximum of two active transmitting antennas per node are used in the transmission at each time slot. The number of receiving antennas at FN as well as the modulation scheme can be arbitrary, and do not affect or complicate the proposed transmitting procedure.

Particularly interesting deployments may be if the frequencies between $\mathrm{SN}$ and RN are reused between RN and FN, or if unlicensed/unauthorized bands are used. 


\section{Conclusions}

In this paper, the new 5G AR transmission scheme with D2D assistance is proposed. In the considered use case it is assumed that source node has unicast stream for far node, and multicast stream for surrounding near users. Relay node is used between SN and FN. Besides unicast stream from SN, RN has another unicast stream for FN. Spatial modulation is implemented at $\mathrm{SN}$ and RN. At the first hop SM is used for multicast streams towards surrounding nodes, while at the second hop SM is used for direct unicast stream towards FN.

The decoding process is not performed at the $\mathrm{RN}$, and the signal is transmitted in the new proposed AF manner. This reduces the complexity of data processing at $\mathrm{RN}$, and saves energy, especially for higher order modulation procedures. Based on proposed processing at the RN, virtual MIMO channel is formed between the SN and FN. In terms of SM at SN/RN, only two antennas are active in each time slot, and the total number of SN/RN antennas can be arbitrary, as only two RF chains are necessary.

Simulation results for BER values are presented, and it can be concluded that there is a possible trade-off between deteriorating BER values and increased spectral/energy efficiency obtained with the proposed scheme.

\section{Declarations}

Funding - Not applicable. Without funding.

Conflicts of interest/Competing interests - Not applicable. Without conflicts of interest/competing interests.

Availability of data and material - Not applicable.

Code availability - Not applicable. 


\section{References}

1. J. Navarro-Ortiz, P. Romero-Diaz, “A Survey on 5G Usage Scenarios and Traffic Models,” IEEE Access, vol. 22, iss. 2, pp. 905 - 929, February 2020.

2. Y. Yang, K. Hua, "Emerging Technologies for 5G-Enabled Vehicular Networks," IEEE Access, vol. 7, pp. 181117 - 181141, November 2019.

3. X. Qiao, P. Ren, "Web AR: A Promising Future for Mobile Augmented Reality—State of the Art, Challenges, and Insights,” Proceedings of the IEEE, vol. 107, iss. 4, pp. 651 - 666, April 2019.

4. X. Ge, L. Pan, "Multipath Cooperative Communications Networks for Augmented and Virtual Reality Transmission,” IEEE Transactions on Multimedia, vol. 19, iss. 10, pp. 2345 - 2358, October 2017.

5. F. Rinaldi, S. Pizzi, “A Novel Approach for MBSFN Area Formation Aided by D2D Communications for eMBB Service Delivery in 5G NR Systems," IEEE Transactions on Vehicular Technology, vol. 69, iss. 2, pp. 2058 - 2070, February 2020.

6. R. I. Ansari, C. Chrysostomou, "5G D2D Networks: Techniques, Challenges, and Future Prospects," IEEE Systems Journal, vol. 12, iss. 4, pp. 3970 - 3984, December 2018.

7. N. Vo, T. Q. Duong, "Optimal Video Streaming in Dense 5G Networks With D2D Communications," IEEE Access, vol. 6, pp. 209 - 223, October 2017.

8. A. Talebi, W.A. Krzymien, "Signal Shaping for Generalized Spatial Modulation and Generalized Quadrature Spatial Modulation,” IEEE Transactions on Wireless Communications, vol. 19, no. 8, pp. 4047 - 4059, June 2019.

9. C. Liu, M. Ma, “Optimal Spatial-Domain Design for Spatial Modulation Capacity Maximization," IEEE Communications Letters, vol. 20, no. 6, pp. 1092 - 1095, April 2016.

10. X. Li, N. Qin, "Interference coordination for FD-MIMO cellular network with D2D communications underlaying," China Communications, vol. 15, iss. 12, pp. 75 - 88, December 2018.

11. A. He, L. Wang, "Spectral and Energy Efficiency of Uplink D2D Underlaid Massive MIMO Cellular Networks,” IEEE Transactions on Communications, vol. 65, iss. 9, pp. 3780 - 3793, June 2017. 
12. B. Ma, H. Shah-Mansouri, "Full-Duplex Relaying for D2D Communication in Millimeter Wave-based 5G Networks,” IEEE Transactions on Wireless Communications, vol. 17, iss. 7, pp. 4417 - 4431, April 2018.

13. S. Dang, G. Chen, "Multicarrier Relay Selection for Full-Duplex Relay-Assisted OFDM D2D Systems,” IEEE Transactions on Vehicular Technology, vol. 67, iss. 8, pp. 7204 - 7218, April 2018.

14. U. Urosevic, Z. Veljovic, M. Pejanovic-Djurisic, "MIMO Solution for Performance Improvements of OFDMCDMA System with Pilot Tone,” Wireless Networks, Volume 19, Issue 8, pp. 2021-2028, November 2013.

15. Z. Veljovic, U. Urosevic, "New Solutions for Cooperative Relaying Implementation of OSTBC with 3/4 Code Rate,” Wireless Personal Communications, Vol. 92, Issue 1, pp. 51-61, Springer, January, 2017.

16. U. Urosevic, Z. Veljovic, “Improving BER performance of virtual QOSTBC,” Wireless Networks, vol. 22, no. 8, pp. 2649-2657, Springer, November 2016.

17. U. Urosevic, Z. Veljovic, M. Pejanovic-Djurisic, “A New Solution for Simple Cooperative Relaying,” Wireless Personal Communications, DOI 10.1007/s11277-013-1420-5, 2013. 